\title{
CT-MR Image Registration in 3D K-Space Based on Fourier Moment Matching
}

\author{
Hong-Ren $\mathrm{Su}^{1}$ and Shang-Hong Lai ${ }^{1,2}$ \\ ${ }^{1}$ Institute of Information Systems and Applications \\ ${ }^{2}$ Department of Computer Science \\ National Tsing Hua University, Hsinchu, Taiwan \\ d9765805@oz.nthu.edu.tw, lai@cs.nthu.edu.tw
}

\begin{abstract}
CT-MRI registration is a common processing procedure for clinical diagnosis and therapy. We propose a novel K-space affine image registration algorithm via Fourier moment matching. The proposed algorithm is based on estimating the affine matrix from the moment relationship between the corresponding Fourier spectrums. This estimation strategy is very robust because the energy of the Fourier spectrum is mostly concentrated in the low-frequency band, thus the moments of the Fourier spectrum are robust against noises and outliers. Our experiments on the real CT and MRI datasets show that the proposed Fourier-based registration algorithm provides higher registration accuracy than the existing mutual information registration technique.
\end{abstract}

Keywords: Multi-modal image registration, Fourier moments, CT, MRI.

\section{Introduction}

Multi-modal medical image registration has been an important research topic in medical imaging due to its great value in clinical applications [1]. One of the most important multi-modal image registrations is the CT-MRI registration [2], which is widely used in minimally invasive surgery and 3D conformal and intensity-modulated radiotherapy (IMRT).

Computed tomography (CT) [3] is commonly used in routine diagnosis since it provides higher spatial accuracy and hard-tissue contrast, such as the bone tissue. It also has the electron density information necessary for radiotherapy (RT) but its disadvantage is the poor soft-tissue contrast, which causes diagnostic problems in soft tissue, like the tumor or nerve. In contrast, magnetic resonance image (MRI) [3] provides superior soft-tissue contrast and visualization in axial, sagittal and coronal planes, allowing better 3D representation for diagnosis. However MRI is not sensitive to the hard tissue and suffers from artifacts at interfaces between bone and air. A feasible and useful strategy [4] for clinical application is to combine the complementary advantages of CT and MRI. Thus, an accurate image registration between CT and MR images is essential for diagnosis and therapy. 
Existing methods used in hospitals for CT-MR registration are mark-and-link [2] or mutual information (MI) based image registration techniques [5]. "Mark-and-link" registration needs matching points between CT and MRI selected by specialists. It spends a lot of money and time but the accuracy is not robust and matching points is not easy selected in a large three dimension data. An automatic and efficient method is the MI technique, which is suitable for multi-modal registration problems and has become a standard algorithm $[6,7,8]$. It is based on minimizing the mutual information cost function between the corresponding voxel image intensities and useful because two modal images have similar intensity distribution. However, MI has an accuracy limitation [4, 8] and cannot always deal with the data well so that many modified methods based on MI have been proposed to improve the accuracy [7].

In this paper, we focus on the CT-MRI registration problem, which is the structure-to-structure image registration. Structure medical images [3], such as CT and MRI, have clear edge information allowing distinguishing one organ area from the others. Thus, there is similar edge information between CT and MRI for human which can be used in the registration. We propose a novel and robust kspace image registration algorithm by using edge information for CT-MRI registration. The K-space is a concept of Fourier space that is well known in medical imaging [9]. The relation between K-space data and image data is the Fourier Transformation.

The K-space registration $[10,11]$ is another intensity-based registration technique that is robust to noise with low computation complexity. It is more accurate and reliable to use the multi-layer fractional Fourier transform [12] than the traditional Fourier transform. However, all the previous image registration methods in K-space can only deal with translation, rotation and scaling, i.e. scaled rigid transformation. In addition, it is not straightforward to extend the K-space registration approach to higherdimensional image registration. Therefore, there has not been much effort of applying the Fourier approach for medical image registration, especially for CT-MRI registration, which involves three-dimensional image registration.

In this paper, we propose a novel K-space affine image registration algorithm for CT-MRI registration. The proposed algorithm is based on the fact that the affine transform between two images corresponds to a related affine transform between their Fourier spectrums [13], whose energies are normally concentrated around the origin in the frequency domain. Thus, the moments for the corresponding Fourier spectrum distributions can be calculated as probability density function. In short, the proposed affine registration algorithm is based on minimizing the affine relationship between the moments for the Fourier spectrums of the two images. In addition, we further extend the algorithm to solve CT and MRI registration problem by representing the $3 \mathrm{D}$ image data as point sets in the 3D space as a binary image. The image registration problem is converted into a point-set registration problem in a $3 \mathrm{D}$ space. We introduce an appropriate distance weighting scheme determined from the shortest distance between the affine transformed point sets in the binary image to achieve better robustness. 
To the best of our knowledge, this is the first work that solves the affine image registration problem by using moment matching in the $\mathrm{K}$-space, and the proposed algorithm can also be applied to higher dimension.

The rest of this paper is organized as follows. In the next section, we briefly review the affine transform relationship between two images in the spatial domain has corresponding affine relationship in the Fourier domain. In section 3, we propose a novel Fourier-based algorithm for robust affine image registration. Experimental results on fMRI motion correction by using the proposed algorithm and some previous methods are given in section 4. Finally, we conclude this paper in the last section.

\section{Fourier-Based Affine Image Registration}

There have been several Fourier-based methods proposed for image registration in the past $[10,11,12,14,15]$. The Fourier-based methods have the advantages that it is efficient and can handle large motion. Recently, some modified Fourier-based image registration methods, like log-polar method [14], multi-layer Fourier transform (MLFFT) [12] and the phase correlation method [15], have been proposed to improve the registration precision and the alignment range. However, all the Fourier-based image registration methods can only deal with rigid transformation and cannot be easily extended to higher dimensions. In this paper, we propose the first Fourier-based affine registration algorithm by estimating the affine transformation from the corresponding Fourier spectrums of the two images.

\section{$2.1 \quad$ Two-Dimensional Case}

Consider two images $\mathrm{g}(\mathrm{x}, \mathrm{y})$ and $\mathrm{h}(\mathrm{x}, \mathrm{y})$ and they are related by an affine transformation [13], i.e. $h(x, y)=g(a x+b y+c, d x+e y+f)$. Assume the Fourier transforms of the image functions $g(x, y)$ and $h(x, y)$ be denoted by $G(u, v)$ and $H(u, v)$, respectively. Then, we can derive the following affine relationship between their Fourier transforms $\mathrm{G}(\mathrm{u}, \mathrm{v})$ and $\mathrm{H}(\mathrm{u}, \mathrm{v})$ given as follows []:

$$
\mathrm{G}(\mathrm{u}, \mathrm{v})=\frac{1}{|\Delta|} e^{i \frac{[(e c-b f) u+(a f-c d) v]}{\Delta}} \mathrm{H}\left(\frac{e u-d v}{\Delta}, \frac{-b u+a v}{\Delta}\right)
$$

By letting $\mathrm{u}^{\prime}=(\mathrm{eu}-\mathrm{dv}) / \Delta$ and $\mathrm{v}^{\prime}=(-\mathrm{bu}+\mathrm{av}) / \Delta$, we have the relationship $\mathrm{u}=\mathrm{au}^{\prime}+\mathrm{dv}^{\prime}$ and $\mathrm{v}=\mathrm{bu}^{\prime}+\mathrm{ev}$ '. Taking the absolute values on both sides of eq. (1), we have the affine transformation relationship between the spectrums $|\mathrm{G}(\mathrm{u}, \mathrm{v})|$ and $|\mathrm{H}(\mathrm{u}, \mathrm{v})|$ as follows:

$$
|\mathrm{G}(\mathrm{u}, \mathrm{v})|=\frac{1}{|\Delta|}\left|H\left(u^{\prime}, v^{\prime}\right)\right|
$$

where

$$
\left[\begin{array}{l}
u \\
\mathrm{v}
\end{array}\right]=\left[\begin{array}{ll}
a & d \\
b & e
\end{array}\right]\left[\begin{array}{l}
u^{\prime} \\
\mathrm{v}^{\prime}
\end{array}\right], \Delta=\left|\begin{array}{ll}
a & b \\
d & e
\end{array}\right|=a e-b d
$$


From eq. (2) and (3), we can see the Fourier spectrums of two images related by an affine transform are also related by the corresponding affine transform. If we can estimate the four affine parameters in eq. (3) from the two Fourier spectrums, the remaining two affine parameters can be computed by transforming image $\mathrm{g}(\mathrm{x}, \mathrm{y})$ with the transformation $\mathrm{g}^{\prime}(\mathrm{x}, \mathrm{y})=\mathrm{g}(\mathrm{ax}+\mathrm{by}, \mathrm{dx}+\mathrm{ey})$ and determining the translation vector $(\mathrm{c}, \mathrm{f})$ between $g^{\prime}(x, y)$ and $h(x, y)$ from their cross power spectrum. To be more specific, the translation vector is determined as follows:

$$
(\mathrm{c}, \mathrm{f})=\arg \max _{(x, y)} \operatorname{real}\left(\operatorname{IFT}\left\{\frac{G^{\prime}(u, v) H^{*}(u, v)}{\left|G^{\prime}(u, v) H^{*}(u, v)\right|}\right\}\right)
$$

where $\mathrm{G}^{\prime}(\mathrm{u}, \mathrm{v})$ is the Fourier transform of $\mathrm{g}^{\prime}(\mathrm{x}, \mathrm{y}), \mathrm{H}^{*}(\mathrm{u}, \mathrm{v})$ is the complex conjugate of $\mathrm{H}(\mathrm{u}, \mathrm{v})$, and IFT denotes the inverse Fourier transform operator.

\subsection{Three-Dimensional Case}

In the above section, the 2D model of the affine transform relationship between image and Fourier domain is described. It is easy to extend it to $3 \mathrm{D}$ or higher dimension model. Consider two 3D image functions $\mathrm{g}(\mathrm{x}, \mathrm{y}, \mathrm{z})$ and $\mathrm{h}(\mathrm{x}, \mathrm{y}, \mathrm{z})$, which are related by an affine transformation, i.e. $h(x, y, z)=g(a x+b y+c z+d, e x+f y+g z+h, i x+j y+k z+1)$, Assume the Fourier transforms of $g(x, y, z)$ and $h(x, y, z)$ be denoted by $G(u, v, w)$ and $\mathrm{H}(\mathrm{u}, \mathrm{v}, \mathrm{w})$, respectively. Then, we can derive the following affine relationship between the Fourier transforms $\mathrm{G}(\mathrm{u}, \mathrm{v}, \mathrm{w})$ and $\mathrm{H}(\mathrm{u}, \mathrm{v}, \mathrm{w})$ given as follows:

$$
|\mathrm{G}(\mathrm{u}, \mathrm{v}, \mathrm{w})|=\frac{1}{|\Delta|}\left|H\left(u^{\prime}, v^{\prime}, w^{\prime}\right)\right|
$$

where

$$
\left[\begin{array}{c}
u \\
\mathrm{v} \\
\mathrm{W}
\end{array}\right]=\left[\begin{array}{lll}
a & e & i \\
\mathrm{~b} & \mathrm{f} & \mathrm{j} \\
\mathrm{c} & \mathrm{g} & \mathrm{k}
\end{array}\right]\left[\begin{array}{l}
u^{\prime} \\
\mathrm{v}^{\prime} \\
\mathrm{w}^{\prime}
\end{array}\right], \Delta=\left|\begin{array}{lll}
a & b & c \\
\mathrm{e} & \mathrm{f} & \mathrm{g} \\
\mathrm{i} & \mathrm{j} & \mathrm{k}
\end{array}\right|
$$

Similar to eq. (4) in the $2 \mathrm{D}$ case, the translation vector $(\mathrm{d}, \mathrm{h}, \mathrm{l})$ between $\mathrm{g}(\mathrm{x}, \mathrm{y}, \mathrm{z})$ and $\mathrm{h}(\mathrm{x}, \mathrm{y}, \mathrm{z})$ can be determined from their cross power spectrums.

From the above discussion on 2D and 3D affine transforms, we can see the main problem to estimate the affine registration parameters based on the Fourier domain approach is how to estimate the affine parameters $(\mathrm{a}, \mathrm{b}, \mathrm{d}, \mathrm{e})$ in the 2D case and $(\mathrm{a}, \mathrm{b}$, $\mathrm{c}, \mathrm{e}, \mathrm{f}, \mathrm{g}, \mathrm{i}, \mathrm{j}, \mathrm{k}$ ) for the 3D case from a pair of Fourier spectrums as given in eq. (2). In this paper, we propose to estimate these affine parameters via the moment matching technique proposed by Ho et al. [16], which will be described subsequently in the next section.

\subsection{Moment Matching Approach to Estimating Affine matrix}

Let the Fourier spectrums of the 2D image functions $f_{1}(x, y)$ and $f_{2}(x, y)$ be denoted by $F_{1}(u, v)$ and $F_{2}(u, v)$, respectively. If $f_{1}(x, y)$ and $f_{2}(x, y)$ are related by an affine transformation, then their Fourier spectrums are also related by the corresponding affine transform, i.e. $\left|\mathrm{F}_{1}(\mathrm{u}, \mathrm{v})\right|=\left|\mathrm{F}_{2}\left(\mathrm{u}^{\prime}, \mathrm{v}^{\prime}\right)\right| / \Delta$ with the relation between $(\mathrm{u}, \mathrm{v})$ 
and $\left(\mathrm{u}^{\prime}, \mathrm{v}^{\prime}\right)$ given in eq. (3). To determine the affine parameters, we employ the moment matching technique to the Fourier spectrums $\left|\mathrm{F}_{1}(\mathrm{u}, \mathrm{v})\right|$ and $\left|\mathrm{F}_{2}(\mathrm{u}, \mathrm{v})\right|$.

The $(\alpha+\beta)$-th moment for the Fourier spectrum $\left|F_{k}(u, v)\right|, k=1$ or 2 , is defined as

$$
\mathrm{m}_{\alpha, \beta}^{\mathrm{k}}=\iint \mathrm{u}^{\alpha} \mathrm{v}^{\beta}\left|\mathrm{F}_{\mathrm{k}}(\mathrm{u}, \mathrm{v})\right| \mathrm{dudv}
$$

By coordinate substitution, we can derive the following equation

$$
\mathrm{m}_{\alpha, \beta}^{2}=\iint\left(a u^{\prime}+d v^{\prime}\right)^{\alpha}\left(b u^{\prime}+e v^{\prime}\right)^{\beta}\left|F_{2}\left(u^{\prime}, v^{\prime}\right)\right| d u^{\prime} d v^{\prime}
$$

Thus, we have the following relationship for the first-order moments.

$$
\left[\begin{array}{l}
\mathrm{m}_{1,0}^{1} \\
\mathrm{~m}_{0,1}^{1}
\end{array}\right]=\left[\begin{array}{ll}
\mathrm{a} & \mathrm{d} \\
\mathrm{b} & \mathrm{e}
\end{array}\right]\left[\begin{array}{l}
\mathrm{m}_{1,0}^{2} \\
\mathrm{~m}_{0,1}^{2}
\end{array}\right]
$$

For the second-order moments, we can derive the following relationship.

$$
\left[\begin{array}{l}
m_{2,0}^{1} \\
m_{1,1}^{1} \\
m_{0,2}^{1}
\end{array}\right]=\left[\begin{array}{ccc}
a^{2} & 2 a d & d^{2} \\
a b & a e+b d & d e \\
b^{2} & 2 b e & e^{2}
\end{array}\right]\left[\begin{array}{l}
m_{2,0}^{2} \\
m_{1,1}^{2} \\
m_{0,2}^{2}
\end{array}\right]
$$

The 2D affine parameters $(\mathrm{a}, \mathrm{b}, \mathrm{d}, \mathrm{e})$ can be estimated by minimizing the total errors associated with the constraints in eq. (9) and (10) in a least-squares estimation framework [16].

The 3D affine parameters ( $a, b, c, e, f, g, i, j, k)$ can be estimated by similar moment matching technique from the moments of the Fourier spectrums $F_{1}(u, v, w)$ and $\mathrm{F}_{2}(\mathrm{u}, \mathrm{v}, \mathrm{w})$. The $(\alpha+\beta+\gamma)$-th moment for the Fourier spectrum $\left|\mathrm{F}_{\mathrm{n}}(\mathrm{u}, \mathrm{v}, \mathrm{w})\right|$ is defined as

$$
\mathrm{m}_{\alpha, \beta, \gamma}^{\mathrm{k}}=\iint \mathrm{u}^{\alpha} \mathrm{v}^{\beta} \mathrm{w}^{\gamma}\left|\mathrm{F}_{\mathrm{k}}(\mathrm{u}, \mathrm{v}, \mathrm{w})\right| \mathrm{dudvdw}
$$

Thus, we have the following relationship for the first-order moments in the Fourier spectrums.

$$
\left[\begin{array}{l}
\mathrm{m}_{1,0,0}^{1} \\
\mathrm{~m}_{0,1,0}^{1} \\
\mathrm{~m}_{0,0,1}^{1}
\end{array}\right]=\left[\begin{array}{lll}
a & e & i \\
\mathrm{~b} & \mathrm{f} & \mathrm{j} \\
\mathrm{c} & \mathrm{g} & \mathrm{k}
\end{array}\right]\left[\begin{array}{l}
\mathrm{m}_{1,0,0}^{2} \\
\mathrm{~m}_{0,1,0}^{2} \\
\mathrm{~m}_{0,0,1}^{2}
\end{array}\right]
$$

For the second-order Fourier moments, we can derive the following relationship.

$$
\left[\begin{array}{l}
\mathrm{m}_{2,0,0}^{1} \\
\mathrm{~m}_{0,2,0}^{1} \\
\mathrm{~m}_{0,0,2}^{1} \\
\mathrm{~m}_{1,1,0}^{1} \\
\mathrm{~m}_{1,0,1}^{1} \\
\mathrm{~m}_{0,1,1}^{1}
\end{array}\right]=\left[\begin{array}{cccccc}
a^{2} & e^{2} & i^{2} & 2 a e & 2 a i & 2 e i \\
b^{2} & f^{2} & j^{2} & 2 b f & 2 b j & 2 f j \\
c^{2} & g^{2} & k^{2} & 2 c g & 2 c k & 2 g k \\
a b & e f & i j & a f+b e & a j+b i & e j+f i \\
a c & e g & i k & a g+c e & a k+c i & e k+g i \\
b c & f g & j k & b g+c f & b g+c f & f k+g j
\end{array}\right]\left[\begin{array}{l}
\mathrm{m}_{2,0,0}^{2} \\
\mathrm{~m}_{0,2,0}^{2} \\
\mathrm{~m}_{0,0,2}^{2} \\
\mathrm{~m}_{1,1,0}^{2} \\
\mathrm{~m}_{1,0,1}^{2} \\
\mathrm{~m}_{0,1,1}^{2}
\end{array}\right]
$$


The relationship of the first-order and second-order Fourier moments, given in eq. (12) and (13), can be used for the least-squares estimation of the above nine 3D affine parameters.

\section{Iterative Refinement Process by a Distance Weighting}

In the previous section, we introduce a novel Fourier-based image registration by applying the moment matching technique to the Fourier spectrums of the image functions. To further improve the accuracy of the novel Fourier-based image registration algorithm, especially in the occlusive problem, we propose an iterative refinement process by introducing a distance weighting scheme into images, which is detailed in the following.

\subsection{Canny Edge Image Data}

Let a point set $\mathrm{p}(\mathrm{x}) \in \mathrm{E}$, which $\mathrm{E}$ is the set of the canny edge, $\mathrm{x} \in \mathrm{R}^{\mathrm{n}}$, is extracted from an image $h(x)$. Then the image $h(x)$ is transferred into a binary image $B(x)$ with values of the pixels corresponding to the points $\mathrm{p}$ set to 1 , and the rest set to 0 .

$$
\mathrm{B}(\mathbf{x})= \begin{cases}1 & (\mathbf{x}) \in \mathrm{p} \\ 0 & (\mathbf{x}) \notin \mathrm{p}\end{cases}
$$

\subsection{Distance Weighting}

The idea to improve the accuracy of the proposed Fourier-based affine image registration is to assign an appropriate weight to each point $\mathrm{p}$ in the binary image such that the points without correspondences have very small weights and the points with proper correspondences have high weights in the binary image. In the previous definition of the binary image B given in eq. (14), the function has binary values to indicate presence of data points. Since the point sets may contain some noise variation, we introduce a distance weighting to reduce the influence of some points without proper correspondences.

The distance for one data point $\mathbf{p} \in \mathrm{E}_{1}$ in the binary image $\mathrm{B}_{1}(\mathbf{x})$ to the other point set $\mathrm{E}_{2}$ for the binary image $\mathrm{B}_{2}(\mathbf{x})$ is defined as

$$
\mathrm{d}\left(\mathrm{p}, \mathrm{E}_{2}\right)=\min _{\mathrm{q} \in \mathrm{E}_{2}}\|\mathrm{p}-\mathrm{q}\|
$$

Note that the distance for all data points in $E_{1}$ to $E_{2}$ can be efficiently computed by using the distance transform. Then, we define the weighting for each data point in $\mathrm{E}_{1}$ to $\mathrm{E}_{2}$ as follows:

$$
\mathrm{w}\left(\mathrm{p}, \mathrm{E}_{2}\right)=\frac{\sigma^{2}}{\sigma^{2}+\mathrm{d}^{2}\left(\mathrm{p}, \mathrm{E}_{2}\right)}
$$

Thus, we can compute the weighting function $\mathrm{Bw}_{1}$ for a binary image $\mathrm{B}_{1}$ as follows: 


$$
\mathrm{Bw}_{1}(\mathrm{x})=\left\{\begin{array}{cc}
\mathrm{w}\left((\mathrm{x}), \mathrm{E}_{2}\right) & (\mathrm{x}) \in \mathrm{E}_{1} \\
0 & (\mathrm{x}) \notin \mathrm{E}_{1}
\end{array}\right.
$$

Similarly, we can compute the weighting function $\mathrm{Bw}_{2}$ for a binary image $\mathrm{B}_{2}$ as follows:

$$
\mathrm{Bw}_{2}(\mathbf{x})=\left\{\begin{array}{cc}
\mathrm{w}\left((\mathbf{x}), \mathrm{E}_{1}\right) & (\mathbf{x}) \in \mathrm{E}_{2} \\
0 & (\mathbf{x}) \notin \mathrm{E}_{2}
\end{array}\right.
$$

In our algorithm, we first apply the Fourier-based affine image registration to the binary image $B_{1}$ and $B_{2}$ without using the distance weighting. The estimated affine transform is applied to all points in $\mathrm{E}_{1}$ to update the point set $\mathrm{E}_{1}$. Then, the distance weighting is computed to produce the weighting functions $\mathrm{Bw}_{1}$ and $\mathrm{Bw}_{2}$ as eq. (17) and (18). The Fourier-based affine image registration algorithm is applied to find the affine transformation between $\mathrm{Bw}_{1}$ and $\mathrm{Bw}_{2}$, and the affine estimation result is used to refine the affine registration. This refinement process is repeated several times until convergence.

The iterative registration is very crucial to the robustness of the registration. If the first step cannot provide robust registration, then the iterative refinement may not converge to the correct registration results. The proposed Fourier-based affine registration algorithm is robust even without the iterative refinement. It is because the Fourier transform of the image bring most of the energy to the low-frequency region in the Fourier domain, thus making the affine registration determined from the moments of the Fourier coefficients robust against noises and outliers.

\subsection{Proposed Affine Image Registration Algorithm with Iterative Refinement}

In this section, we summarize the proposed affine image registration algorithm. The detailed procedure is given as follows:

1. Generate the Canny edge as binary images $B_{1}$ and $B_{2}$ from the two images $\mathrm{h}(\mathbf{x})$ and $\mathrm{g}(\mathbf{x})$ as eq. (14).

2. Compute the discrete Fourier transforms of $\mathrm{B}_{1}$ and $\mathrm{B}_{2}$ via FFT.

3. Compute the first-order and second-order moments for the amplitude in Fourier spectrums of $\mathrm{B}_{1}$ and $\mathrm{B}_{2}$ from eq. (11)

4. Determine the affine parameters in matrix $A$ by minimizing the least-square errors associated with the moment matching constraints given in eq. (12) and (13).

5. Transform $B_{1}$ with the affine transform with the estimated matrix $A$ and the transformed data is denoted by $B_{1}{ }^{\prime}$.

6. Determine the translation vector $\mathrm{t}$ between $B_{1}{ }^{\prime}$ and $\mathrm{B}_{2}$ via the cross power spectrum method given in eq. (4).

7. Shift the map $B_{1}{ }^{\prime}$ with the translation vector $\mathrm{t}$ and compute the distance weighting from eq. (17) to form the weighting function $\mathrm{Bw}_{1}$.

8. Repeat step 2 to step 7 with the binary image replaced by the weighting functions computed in the previous step until the changes in the affine transformation parameters are within a small threshold.

Note that the above affine image registration algorithm can be used in any highdimensional image data. 


\section{Experimental Results}

In our experiments, the CT and MRI 3D datasets in Vanderbilt Database [17], from the Retrospective Image Registration Evaluation (RIRE) project, are used for comparing the registration accuracy of different methods in CT to MRI registration. In our experiment, we compare the proposed Fourier-moment based image registration with the image registration program used in SPM8 [18] which is based on mutual information registration [19]. In the Vanderbilt Database, each patient has four 3D data sets, such as CT $(512 \times 512 \times 28)$, MR-T1 (256x256x25), MR-T2 (256x256x25) and MR-PD (256x256x25). The data sets also provide manually annotated eight corresponding points for CT to MR-T1, CT to MR-T2 and CT to MR-PD. We calculate the average distances of the eight corresponding points in the image data after the CT-MR registration as the performance measure.

Table 1. Average distances of eight corresponding points between original data to the data after CR-MR registration

\begin{tabular}{ccc}
\hline CT-MR registration & Proposed method & SPM8[18, 19] \\
\hline CT to MR-PD & $0.80 \mathrm{~mm}$ & $0.89 \mathrm{~mm}$ \\
CT to MR-T1 & $0.76 \mathrm{~mm}$ & $0.83 \mathrm{~mm}$ \\
CT to MR-T2 & $0.93 \mathrm{~mm}$ & $1.07 \mathrm{~mm}$ \\
\hline
\end{tabular}

The proposed method is used for 3D CT-MR registration. Fig. 1 and 2 depict some examples of the images, and the corresponding edge maps and K-space representations. Fig. 1 shows the multi-modal images with their edge maps by Canny edge detection and the corresponding K-spaces. Table 1 shows the proposed method has smaller distances between the corresponding landmark points after registration than the mutual information based algorithm [19] used in SPM8 [18].

Fig. 2 shows the edge maps in one axial plane after CT-MR registration by using the proposed algorithm. CT image has higher resolution than MR image so that the CT-MR registration problem is not only a 3D multi-modal registration but also a 3D muti-resolution registration. We can see from Fig. 2 that the experiments for CT to MR-PD and CT to MR-T1 have better registration results.

For simulated affine image registration experiment, we used the brain atlas MRI data set from [20] and generate synthesized images with 50 random affine transformations from the T1 and T2 brain images. Some sample images used in this experiment are shown in Fig. 3. The average relative errors in affine registration obtained by using the proposed algorithm and the affine registration tool in the SPM software [18], which is based on the algorithm proposed in [19], are evaluated and shown in Table 2.

Note that the relative error for the affine matrix estimation is defined as

$$
\text { Relative error }=\|\widehat{A}-\mathrm{A}\|_{\mathrm{F}} /\|\mathrm{A}\|_{\mathrm{F}}
$$




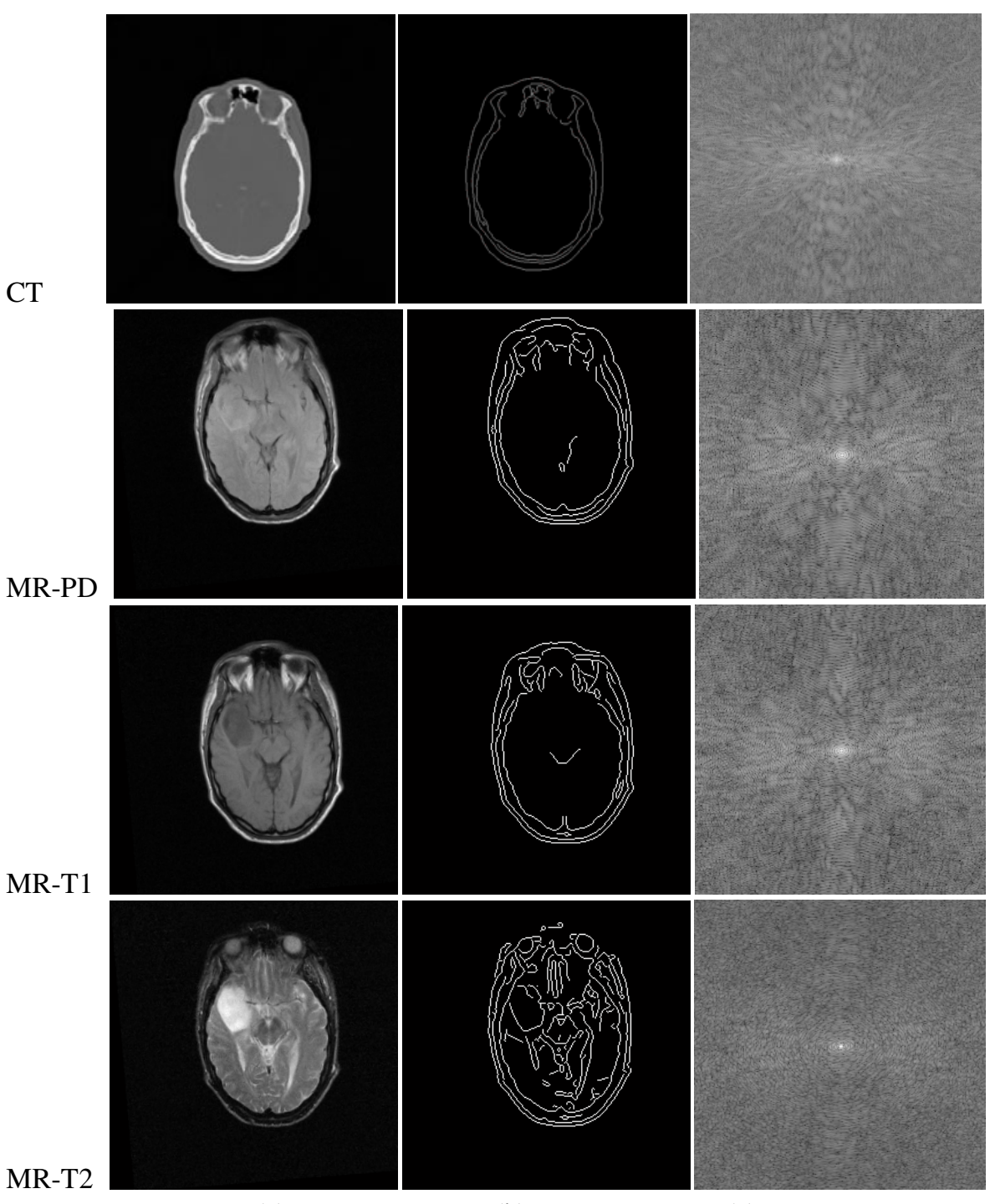

(a)

(b)

(c)

Fig. 1. Example images used in the CT-MR registration experiment from Vanderbilt Database: (a) original brain CT and MRI images, (b) canny edge maps from (a), (c) K-spaces computed from (b) 

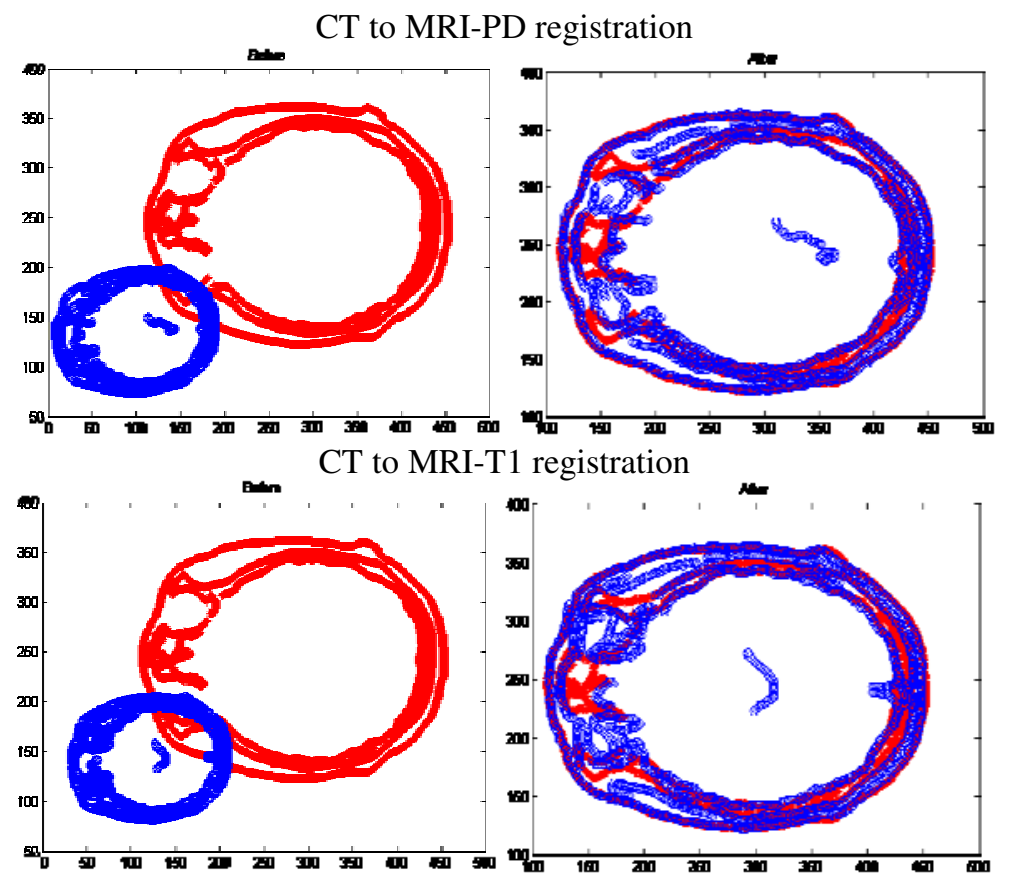

CT to MRI-T2 registration

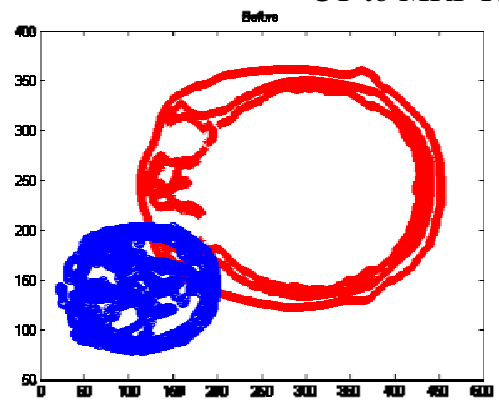

(a)

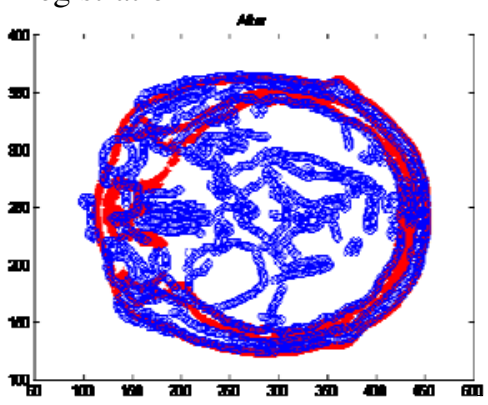

(b)

Fig. 2. Example images from an axial plane used in the CT-MR registration experiment from Vanderbilt Database: overlay of the two corresponding edge maps (a) before registration and (b) after registration by using the proposed algorithm

where $\mathrm{A}$ is the ground-truth $3-$ by-3 affine matrix, $\hat{\mathbf{A}}$ is the estimated affine matrix, and \|\|$_{F}$ denotes the Frobenius norm for a matrix.

Table 2. Average relative errors in the image registration experiment by using the proposed algorithm and SPM [18]

\begin{tabular}{ccc}
\hline $\begin{array}{c}\text { MR T1-T2 } \\
\text { registration }\end{array}$ & The proposed method & SPM8[18, 19] \\
\hline MR-T1 to MR-T2 & 0.14 & 0.26 \\
\hline
\end{tabular}




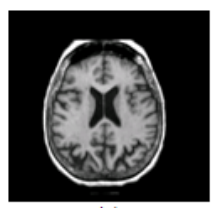

(a)

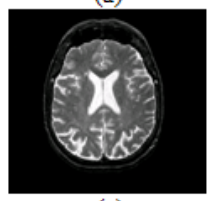

(c)
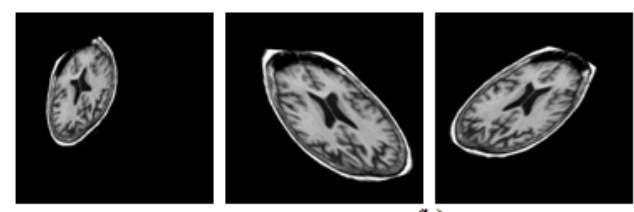

(b)

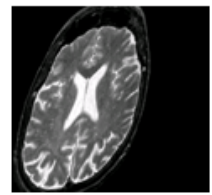

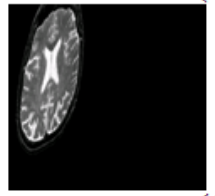

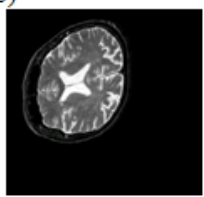

(d)
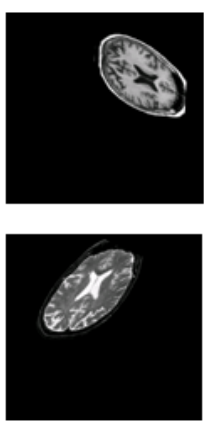

Fig. 3. Example images used in the affine image registration experiment: (a) original $\mathrm{T} 1$ brain MRI image, (b) synthesized images from (a) with random affine transforms, (c) original T2 brain MR image, (d) synthesized images from (c) with random affine transforms

\section{Conclusions}

In this paper, we proposed a robust affine registration algorithm in k-space that can be applied to both image alignment and multi-modal CT-MR registration. The proposed algorithm is based on matching the moments of the Fourier spectrums for two point distributions to alleviate the sensitivity problem of the moment-based approach. To further improve the robustness of the proposed algorithm, we proposed to incorporate the distance weighting to the canny edge for iterative refinement of the affine registration. Our experiments demonstrated the superior performance of the proposed affine image registration algorithm which is suitable for CT-MR registration. In the future, we aim to extend this robust affine registration algorithm to overcome more challenging multi-modal medical image registration problems.

\section{References}

1. Hawkes, D.J.: Algorithms for radiological image registration and their clinical application. Journal of Anatomy 193(3), 347-361 (1998)

2. XiaoShen, W., LongGen, L., ChaoSu, H., JianJian, Q., ZhiYong, X., Yan, F.: A comparative study of three CT and MRI registration algorithms in nasopharyngeal carcinoma. Journal of Applied Clinical Medical Physics 10(2) (2009)

3. Jean-François, D., Mérence, S., Anne, B., Guy, C., Max, L., Vincent, G.: Evaluation of a multimodality image (CT, MRI and PET) coregistration procedure on phantom and head and neck cancer patients: accuracy, reproducibility and consistency. Radiotherapy \& Oncology 69(3), 237-245 (2003)

4. Antoine Maintz, J.B., Viergever, M.A.: A survey of medical image registration. Medical Image Analysis 2(1), 1-36 (1998)

5. Zitová, B., Flusser, J.: Image registration methods: a survey. Image Vision Computing 21(11), 977-1000 (2003) 
6. West, J., et al.: Comparison and evaluation of retrospective inter-modality brain image registration techniques. Journal of Computer Assisted Tomography 21(4), 554-566 (1997)

7. Josien, P.W., Pluim, J.B., Antoine, M., Max, A.V.: Mutual information based registration of medical images: a survey. IEEE Trans. Med. Imaging 22(8), 986-1004 (2003)

8. Maes, F., Collignon, A., Vandermeulen, D., Marchal, G., Suetens, P.: Multimodality image registration by maximization of mutual information. IEEE Transactions on Medical Imaging 16(2), 187-198 (1997)

9. Twieg, D.: The k-trajectory formulation of the NMR imaging process with applications in analysis and synthesis of imaging methods. Medical Physics 10(5), 610-621 (1983)

10. De Castro, E., Morandi, C.: Registration of translated and rotated images using finite Fourier transforms. IEEE Trans. Pattern Analysis Mach. Intell. 3, 700-703 (1987)

11. Reddy, B.S., Chatterji, B.N.: An FFT-based technique for translation, rotation, and scaleinvariant image registration. IEEE Trans. Pattern Analysis Mach. Intell. 5(8), 1266-1270 (1996)

12. Pan, W., Qin, K., Chen, Y.: An adaptable-multilayer fractional Fourier transform approach for image registration. IEEE Trans. Pattern Analysis Mach. Intell. 31(3), 400-413 (2009)

13. Bracewell, R.N., Chang, K.Y., Jha, A.K., Wang, Y.H.: Affine theorem for twodimensional Fourier transform. Electronics Letters 29(3), 304 (1993)

14. Foroosh, H., Zerubia, J.B., Berthod, M.: Extension of phase correlation to subpixel registration. IEEE Trans. Image Processing 11(3), 188-200 (2002)

15. Zokai, S., Wolberg, G.: Image registration using log-polar mappings for recovery of largescale similarity and projective transformations. IEEE Trans. Image Processing 14(10), 1422-1434 (2005)

16. Ho, J., Peter, A., Ranganrajan, A., Yang, M.-H.: An algebraic approach to affine registration of point sets. Proc. Int. Conf. on Computer Vision 2009 (2009)

17. Vanderbilt Database, http://www. insight-journal.org/rire/index.php

18. SPM, http://www.fil.ion.ucl.ac.uk/spm/

19. D’Agostino, E., Maes, F., Vandermeulen, D., Suetens, P.: Non-rigid atlas-to-image registration by minimization of class-conditional image entropy. In: Barillot, C., Haynor, D.R., Hellier, P. (eds.) MICCAI 2004, Part I. LNCS, vol. 3216, pp. 745-753. Springer, Heidelberg (2004)

20. The Whole Brain Atlas, http: / /www . med. harvard. edu / AANLIB / home. html 